Document downloaded from:

http://hdl.handle.net/10251/64601

This paper must be cited as:

Hernández, AE.; Lattanzi, MB.; Thome, N. (2015). Weighted binary relations involving the Drazin inverse. Applied Mathematics and Computation. 253:215-223. doi:10.1016/j.amc.2014.12.102.

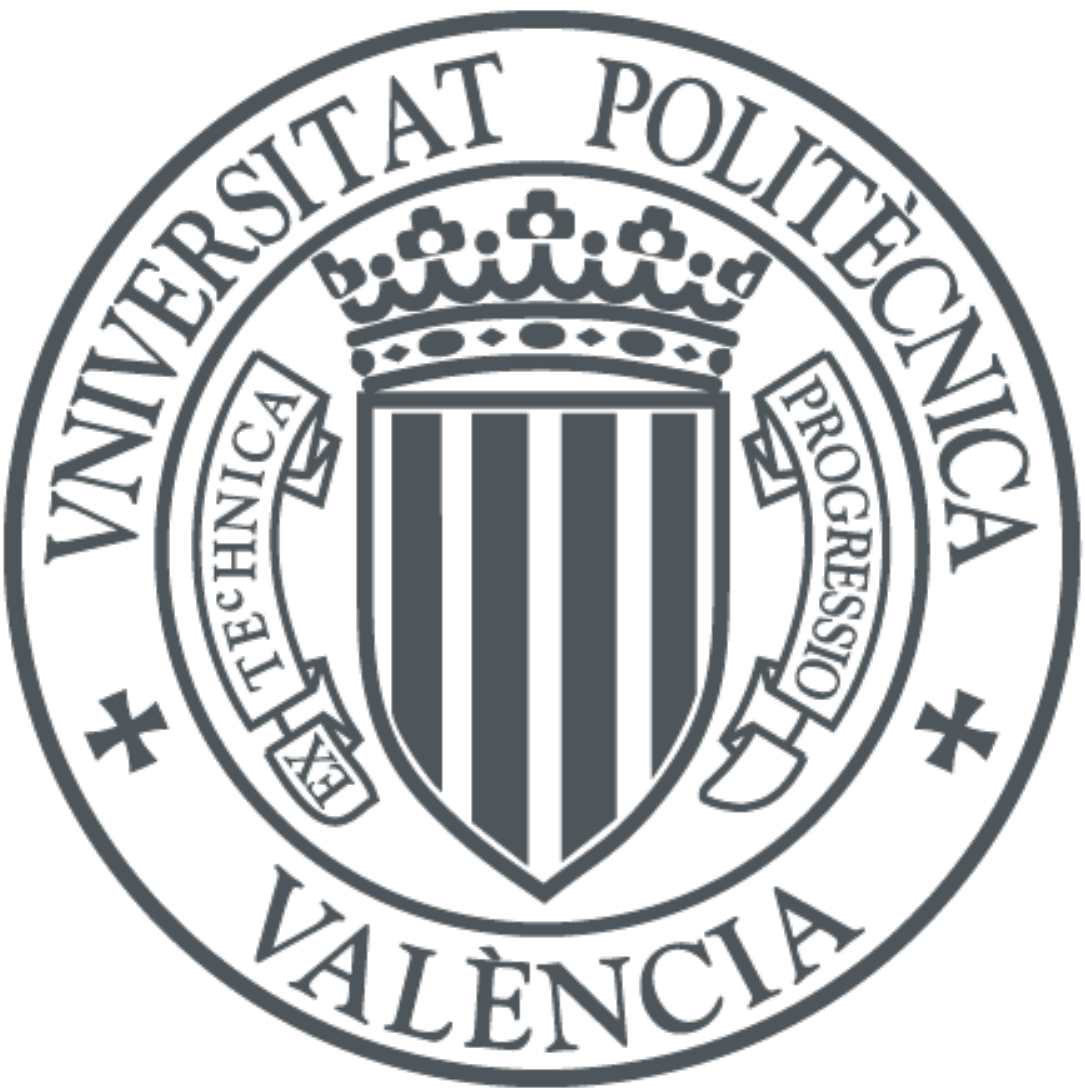

The final publication is available at

https://dx.doi.org/10.1016/j.amc.2014.12.102

Copyright Elsevier

Additional Information 


\title{
Weighted binary relations involving the Drazin inverse
}

\author{
A. Hernández ${ }^{* \dagger} \quad$ M. Lattanzi ${ }^{\dagger} \quad$ N. Thome ${ }^{\ddagger}$
}

\begin{abstract}
The Drazin inverse of a matrix has been used in the literature to define a preorder on the set of square complex matrices. In this paper we analyze new binary relations defined on the set of rectangular complex matrices and some relationships to the $W$-support idempotent. We introduce the class of weighted Drazin equal projectors and analyze the pre-orders on this class. Moreover, adjacent matrices are studied under the considered relations. Finally, some observations on weighted partial orders are given.
\end{abstract}

AMS Classification: 15A09, 06A06

Keywords: Drazin inverse; Drazin pre-order; sharp partial order.

\section{Introduction}

The symbol $\mathbb{C}^{m \times n}$ denotes the set of $m \times n$ complex matrices. For a given $A \in \mathbb{C}^{m \times n}$, the notation $A^{*}$ stands for the conjugate transpose of $A$. As usual, $I_{n}$ and $O_{n}$ denote the $n \times n$ identity and zero matrices, respectively. The subscripts will be deleted when no confusion is caused. Given two matrices $A \in \mathbb{C}^{t \times t}$ and $B \in \mathbb{C}^{(m-t) \times(n-t)}$ we will denote

*Facultad de Ingeniería, Universidad Nacional de La Pampa, Calle 110 No 390, General Pico, La Pampa. Argentina. E-mail: aracelih@ing.unlpam.edu.ar.

${ }^{\dagger}$ Facultad de Ciencias Exactas y Naturales, Universidad Nacional de La Pampa, Av. Uruguay 151, Santa Rosa, La Pampa. Argentina. E-mail: mblatt@exactas.unlpam.edu.ar .

¥Instituto Universitario de Matemática Multidisciplinar, Universitat Politècnica de València, 46022, València, Spain. E-mail: njthome@mat.upv.es . 
by $A \oplus B$ the $m \times n$ matrix where $A$ is in the $\mathrm{N}-\mathrm{W}$ corner, $B$ is in the $\mathrm{S}$-E corner and the other two blocks correspond to rectangular zero matrices of adequate sizes.

Let $A \in \mathbb{C}^{n \times n}$. The index of $A$, denoted by $\operatorname{ind}(A)$, is the smallest nonnegative integer $k$ such that $A^{k}$ and $A^{k+1}$ have the same rank. The only matrix $X \in \mathbb{C}^{n \times n}$ satisfying $X A X=X, A X=X A$, and $A^{k+1} X=A^{k}$, with $k=i n d(A)$, is called the Drazin inverse of $A$ [3]. It always exists and is denoted by $X=A^{D}$. It is clear that $A^{r+1} A^{D}=A^{r}$, for all integer $r \geq \operatorname{ind}(A)$ and $A^{r+1} A^{D}=A^{D} A^{r+1}$, for all integer $r \geq 0$. We recall that if $A$ has index at most 1 , the Drazin inverse of $A$ is called the group inverse of $A$ and is denoted by $A^{\#}$. The Drazin inverse of a matrix can be computed via the core-nilpotent decomposition. Indeed, for a nonzero given matrix $A \in \mathbb{C}^{n \times n}$ with $\operatorname{ind}(A)=k$, there exist nonsingular matrices $P \in \mathbb{C}^{n \times n}$ and $C \in \mathbb{C}^{a \times a}$ such that $A=P(C \oplus N) P^{-1}$ where $N \in \mathbb{C}^{(n-a) \times(n-a)}$ is absent $(k=0), N=O_{n-a}(k=1)$ or $N \in \mathbb{C}^{(n-a) \times(n-a)}$ is a nonzero nilpotent matrix with nilpotence index equals $k>1$. By abuse of language, we will say that $N$ is nilpotent for each one of these three possibilities. In this case, $A^{D}=P\left(C^{-1} \oplus O\right) P^{-1}$. We can write $A$ in the core-nilpotent decomposition as $A=A_{1}+A_{2}$ where $A_{1}=P(C \oplus O) P^{-1}$ and $A_{2}=P(O \oplus N) P^{-1}$ and $A_{1}, A_{2}$ are the unique matrices in these conditions (see [3]).

The following result will be used in the sequel.

Theorem 1.1 [4, Lemma 1.1] If $W \in \mathbb{C}^{n \times m}$ is a nonzero matrix, $A \in \mathbb{C}^{m \times n}, k_{1}=$ $\operatorname{ind}(A W)$, and $k_{2}=\operatorname{ind}(W A)$ then there exist four nonsingular matrices $P \in \mathbb{C}^{m \times m}$, $Q \in \mathbb{C}^{n \times n}, A_{1}, W_{1} \in \mathbb{C}^{t \times t}$, and two matrices $A_{2} \in \mathbb{C}^{(m-t) \times(n-t)}$ and $W_{2} \in \mathbb{C}^{(n-t) \times(m-t)}$ such that $A_{2} W_{2}$ and $W_{2} A_{2}$ are nilpotent of indices $k_{1}$ and $k_{2}$, respectively, where

$$
A=P\left(A_{1} \oplus A_{2}\right) Q^{-1} \quad \text { and } \quad W=Q\left(W_{1} \oplus W_{2}\right) P^{-1}
$$

We observe that Theorem 1.1 can also be established for $k=\max \left\{k_{1}, k_{2}\right\}$. Moreover, the matrix $W$ can be seen as a weight needed to transform the rectangular matrix $A$ into two square ones, namely, $A W$ and $W A$.

On the other hand, we recall that a binary relation is a pre-order if it is reflexive and transitive and, a partial order, if it is also antisymmetric. Partial orders have been widely studied (see, for example, [8] and the references therein). Interesting applications of partial orders and pre-orders were investigated, for instance, in [1,2]. In those papers, properties on the distribution of quadratics forms in normal variables are dealt in the 
Cochran's Theorem environment. The utility of a pre-order was studied, for instance, in [1]. The authors generalized a property on the independence of two quadratic forms that involves the Löwner partial order (i.e., $A \leq_{L} B$ if $B-A$ is nonnegative definite where $A$ and $B$ are $n \times n$ symmetric matrices), replacing $\leq_{L}$ by the column space pre-order, which is simpler to be verified. For more applications, we refer the reader to [12].

The following binary relations are well known.

Let $A, B \in \mathbb{C}^{n \times n}$ be matrices with index at most 1 . It is said that $A$ is below $B$ under the sharp partial order, and is denoted by $A \leq^{\#} B$, if $A^{\#} A=A^{\#} B$ and $A A^{\#}=B A^{\#}$.

Suppose that $A, B \in \mathbb{C}^{n \times n}$ are matrices of arbitrary index, and they are written in the respective core-nilpotent decompositions as $A=A_{1}+A_{2}$ and $B=B_{1}+B_{2}$. It is said that $A$ is related to $B$ under the Drazin pre-order, and is denoted by $A \preceq^{d} B$, if $A_{1} \leq^{\#} B_{1}$. Observe that $A \preceq^{d} B$ is equivalent to $A^{D} A=A^{D} B$ and $A A^{D}=B A^{D}$.

The main aim of this paper is to investigate some new binary relations defined on the set of rectangular matrices $\mathbb{C}^{m \times n}$.

This paper is organized as follows. Section 2 introduces and characterizes three relations considered on rectangular matrices: $\preceq^{d, W, r}, \preceq^{d, W, \ell}$, and $\preceq^{d, W}$. Moreover, the concept of $W$-support idempotent is recalled and some links to these relations are given. For each rectangular matrix $A$ and a fixed weight $W$, it is possible to define two projectors involving the Drazin inverse of $A W$ and $W A$. Section 3 analyzes the class of all matrices for which those two projectors coincide. Additionally, the relation $\preceq^{d, W}$ is studied on this class. In Section 4, we characterize the adjacent matrices under the three considered relations. Finally, in Section 5, some considerations on weighted partial orders are given.

\section{Weighted binary relations and the Drazin inverse}

The fact that Drazin inverse exists for all square matrix allowed to define the Drazin pre-order on square matrices [8]. However, it is not possible to define this pre-order for rectangular matrices in the same way. In order to do that, we are going to consider a weight matrix and define some binary relations on the set of rectangular matrices by means of the Drazin inverse of certain square matrices.

Definition 2.1 Let $W \in \mathbb{C}^{n \times m}$ a nonzero matrix and $A, B \in \mathbb{C}^{m \times n}$. It is said that 
(a) $A \preceq^{d, W, r} B$ if $A W \preceq^{d} B W$,

(b) $A \preceq^{d, W, \ell} B$ if $W A \preceq^{d} W B$,

(c) $A \preceq^{d, W} B$ if $A \preceq^{d, W, r} B$ and $A \preceq^{d, W, \ell} B$,

where $\preceq^{d}$ is adequately considered on $\mathbb{C}^{m \times m}$ or $\mathbb{C}^{n \times n}$.

Using that $\preceq^{d}$ is a pre-order we obtain the following result.

Lemma 2.1 The binary relations $\preceq^{d, W, r}, \preceq^{d, W, \ell}$, and $\preceq^{d, W}$ define a pre-order on $\mathbb{C}^{m \times n}$.

Next we characterize the relation $\preceq^{d, W, r}$ in terms of block decompositions of the involved matrices. Before doing that, notice that $\preceq^{d, W, r}, \preceq^{d, W, \ell}$, and $\preceq^{d, W}$ pre-orders do not preserve equivalences (that is, in general that $A \preceq{ }^{\diamond} B$ implies $\Gamma_{1} A \Gamma_{2} \preceq^{\diamond} \Gamma_{1} B \Gamma_{2}$ is not valid for all nonsingular $\Gamma_{1}, \Gamma_{2}$ and for each $\left.\preceq^{\diamond} \in\left\{\preceq^{d, W, r}, \preceq^{d, W, \ell}, \preceq^{d, W}\right\}\right)$. In order to illustrate this situation we give the following example.

Example 2.1 The matrices

$A=\left[\begin{array}{lll}1 & 0 & 1 \\ 0 & 1 & 0\end{array}\right], B=\left[\begin{array}{lll}0 & 0 & 1 \\ 1 & 1 & 0\end{array}\right], W=\left[\begin{array}{ll}0 & 0 \\ 0 & 1 \\ 1 & 0\end{array}\right], \Gamma_{1}=I_{2}, \quad$ and $\Gamma_{2}=\left[\begin{array}{lll}2 & 0 & 1 \\ 0 & 2 & 0 \\ 0 & 0 & 2\end{array}\right]$

satisfy $A W=B W=I_{2}$; however $\Gamma_{1} A \Gamma_{2} \npreceq^{d, W, r} \Gamma_{1} B \Gamma_{2}$ because $\left(\Gamma_{1} A \Gamma_{2} W\right)\left(\Gamma_{1} A \Gamma_{2} W\right)^{D}=$ $I_{2}$ and

$$
\left(\Gamma_{1} B \Gamma_{2} W\right)\left(\Gamma_{1} A \Gamma_{2} W\right)^{D}=\left[\begin{array}{cc}
\frac{2}{3} & 0 \\
\frac{1}{3} & 1
\end{array}\right] .
$$

This fact tells us that we can not remove matrices $P$ and $Q$ when using Theorem 1.1 for characterizing the pre-orders of Lemma 2.1.

Theorem 2.1 Let $W \in \mathbb{C}^{n \times m}$ be a nonzero matrix and $A, B \in \mathbb{C}^{m \times n}$. The following conditions are equivalent:

(a) $A \preceq^{d, W, r} B$.

(b) $(A W)^{D}(A W)=(A W)^{D}(B W)=(B W)(A W)^{D}$. 
(c) $(A W)^{k_{1}}(B W)=(B W)(A W)^{k_{1}}=(A W)^{k_{1}+1}$, where $k_{1}=\operatorname{ind}(A W)$.

(d) There exist nonsingular matrices $P \in \mathbb{C}^{m \times m}, Q \in \mathbb{C}^{n \times n}, A_{1}, W_{1}^{A} \in \mathbb{C}^{t_{A} \times t_{A}}$, and $\left(B_{2}\right)_{1},\left(W_{2}^{A}\right)_{1} \in \mathbb{C}^{t \times t}$, and there exist matrices $A_{2} \in \mathbb{C}^{\left(m-t_{A}\right) \times\left(n-t_{A}\right)}, B_{3} \in \mathbb{C}^{t_{A} \times\left(n-t_{A}\right)}$, $\left(B_{2}\right)_{2} \in \mathbb{C}^{\left(m-t_{A}-t\right) \times\left(n-t_{A}-t\right)}$, and $\left(W_{2}^{A}\right)_{2} \in \mathbb{C}^{\left(n-t_{A}-t\right) \times\left(m-t_{A}-t\right)}$ satisfying

$$
\begin{gathered}
A=P\left(A_{1} \oplus A_{2}\right) Q^{-1}, \quad B=P\left[\begin{array}{cc}
A_{1} & B_{3} \\
O & \left(B_{2}\right)_{1} \oplus\left(B_{2}\right)_{2}
\end{array}\right] Q^{-1}, \\
W=Q\left(W_{1}^{A} \oplus\left(\left(W_{2}^{A}\right)_{1} \oplus\left(W_{2}^{A}\right)_{2}\right)\right) P^{-1},
\end{gathered}
$$

where $A_{2}\left(\left(W_{2}^{A}\right)_{1} \oplus\left(W_{2}^{A}\right)_{2}\right),\left(\left(W_{2}^{A}\right)_{1} \oplus\left(W_{2}^{A}\right)_{2}\right) A_{2},\left(B_{2}\right)_{2}\left(W_{2}^{A}\right)_{2}$, and $\left(W_{2}^{A}\right)_{2}\left(B_{2}\right)_{2}$ are nilpotent matrices and $B_{3}\left(\left(W_{2}^{A}\right)_{1} \oplus\left(W_{2}^{A}\right)_{2}\right)=O$.

Proof. Items (a), (b) and (c) are equivalent taking into account the equality of the projectors $(A W)(A W)^{D}$ and $(A W)^{D}(A W)$ and using the definition of Drazin inverse.

(b) $\Longrightarrow(\mathrm{d})$ Suppose that $A, B \in \mathbb{C}^{m \times n}$ satisfy (b). By Theorem 1.1, there are nonsingular matrices $P_{A} \in \mathbb{C}^{m \times m}, Q_{A} \in \mathbb{C}^{n \times n}, A_{1}, W_{1}^{A} \in \mathbb{C}^{t_{A} \times t_{A}}$, and matrices $A_{2}^{\prime} \in$ $\mathbb{C}^{\left(m-t_{A}\right) \times\left(n-t_{A}\right)}, W_{2}^{A} \in \mathbb{C}^{\left(n-t_{A}\right) \times\left(m-t_{A}\right)}$ satisfying

$$
A=P_{A}\left(A_{1} \oplus A_{2}^{\prime}\right) Q_{A}^{-1}, \quad W=Q_{A}\left(W_{1}^{A} \oplus W_{2}^{A}\right) P_{A}^{-1},
$$

where $A_{2}^{\prime} W_{2}^{A}$ and $W_{2}^{A} A_{2}^{\prime}$ are $k_{1}$-nilpotent and $k_{2}$-nilpotent, respectively, where $k_{1}=$ $\operatorname{ind}(A W)$ and $k_{2}=\operatorname{ind}(W A)$.

Now, we consider the following partition of $B$

$$
B=P_{A}\left[\begin{array}{cc}
B_{1} & B_{3}^{\prime} \\
B_{4} & B_{2}
\end{array}\right] Q_{A}^{-1}
$$

according to the size of the blocks of $A$. Then,

$$
B W=P_{A}\left[\begin{array}{cc}
B_{1} W_{1}^{A} & B_{3}^{\prime} W_{2}^{A} \\
B_{4} W_{1}^{A} & B_{2} W_{2}^{A}
\end{array}\right] P_{A}^{-1}
$$

and using the equality $(A W)^{D}=P_{A}\left(\left(A_{1} W_{1}^{A}\right)^{-1} \oplus O\right) P_{A}^{-1}$ we get

$$
(B W)(A W)^{D}=P_{A}\left[\begin{array}{cc}
B_{1} A_{1}^{-1} & O \\
B_{4} A_{1}^{-1} & O
\end{array}\right] P_{A}^{-1},
$$




$$
(A W)^{D}(B W)=P_{A}\left[\begin{array}{cc}
\left(A_{1} W_{1}^{A}\right)^{-1} B_{1} W_{1}^{A} & \left(A_{1} W_{1}^{A}\right)^{-1} B_{3}^{\prime} W_{2}^{A} \\
O & O
\end{array}\right] P_{A}^{-1},
$$

and $(A W)^{D}(A W)=P_{A}\left(I_{t_{A}} \oplus O\right) P_{A}^{-1}$. From (b), we obtain $B_{1}=A_{1}, B_{4}=O$, and $B_{3}^{\prime} W_{2}^{A}=O$, that is

$$
B=P_{A}\left[\begin{array}{cc}
A_{1} & B_{3}^{\prime} \\
O & B_{2}
\end{array}\right] Q_{A}^{-1}
$$

Suppose that $W_{2}^{A} \neq O$. Applying Theorem 1.1 to matrices $B_{2} \in \mathbb{C}^{\left(m-t_{A}\right) \times\left(n-t_{A}\right)}$ and $W_{2}^{A} \in \mathbb{C}^{\left(n-t_{A}\right) \times\left(m-t_{A}\right)}$, there exist nonsingular matrices $R \in \mathbb{C}^{\left(m-t_{A}\right) \times\left(m-t_{A}\right)}, S \in$ $\mathbb{C}^{\left(n-t_{A}\right) \times\left(n-t_{A}\right)},\left(B_{2}\right)_{1},\left(W_{2}^{A}\right)_{1} \in \mathbb{C}^{t \times t}$, and matrices $\left(B_{2}\right)_{2} \in \mathbb{C}^{\left(m-t_{A}-t\right) \times\left(n-t_{A}-t\right)},\left(W_{2}^{A}\right)_{2} \in$ $\mathbb{C}^{\left(n-t_{A}-t\right) \times\left(m-t_{A}-t\right)}$, satisfying

$$
B_{2}=R\left(\left(B_{2}\right)_{1} \oplus\left(B_{2}\right)_{2}\right) S^{-1} \quad \text { and } \quad W_{2}^{A}=S\left(\left(W_{2}^{A}\right)_{1} \oplus\left(W_{2}^{A}\right)_{2}\right) R^{-1},
$$

where $\left(B_{2}\right)_{2}\left(W_{2}^{A}\right)_{2}$ and $\left(W_{2}^{A}\right)_{2}\left(B_{2}\right)_{2}$ are nilpotent.

Consider the matrices $P \in \mathbb{C}^{m \times m}$ and $Q \in \mathbb{C}^{n \times n}$ defined by

$$
P=P_{A}\left(I_{t_{A}} \oplus R\right) \quad \text { and } \quad Q=Q_{A}\left(I_{t_{A}} \oplus S\right) .
$$

Replacing (2) and (3) in the expressions of $A, B$, and $W$ and setting $A_{2}=R^{-1} A_{2}^{\prime} S$ and $B_{3}=B_{3}^{\prime} S$ we arrive at

$$
A=P\left(A_{1} \oplus A_{2}\right) Q^{-1}, \quad B=P\left[\begin{array}{cc}
A_{1} & B_{3} \\
O & R^{-1} B_{2} S
\end{array}\right] Q^{-1},
$$

and

$$
W=Q\left(W_{1}^{A} \oplus S^{-1} W_{2}^{A} R\right) P^{-1} .
$$

Observe that the case $W_{2}^{A}=O$ can also be written as in (d) with $\left(W_{2}^{A}\right)_{1}=O$ and $\left(W_{2}^{A}\right)_{2}=O$.

(d) $\Longrightarrow$ (b) It is straightforward.

A similar characterization is established for the inequality $\preceq^{d, W, \ell}$.

Theorem 2.2 Let $W \in \mathbb{C}^{n \times m}$ be a nonzero matrix and $A, B \in \mathbb{C}^{m \times n}$. The following conditions are equivalent: 
(a) $A \preceq^{d, W, \ell} B$.

(b) $(W A)^{D}(W A)=(W A)^{D}(W B)=(W B)(W A)^{D}$.

(c) $(W A)^{k_{2}}(W B)=(W B)(W A)^{k_{2}}=(W A)^{k_{2}+1}$, where $k_{2}=i n d(W A)$.

(d) There exist nonsingular matrices $P \in \mathbb{C}^{m \times m}, Q \in \mathbb{C}^{n \times n}, A_{1}, W_{1}^{A} \in \mathbb{C}^{t_{A} \times t_{A}}$, and $\left(B_{2}\right)_{1},\left(W_{2}^{A}\right)_{1} \in \mathbb{C}^{t \times t}$, and there exist matrices $A_{2} \in \mathbb{C}^{\left(m-t_{A}\right) \times\left(n-t_{A}\right)}, B_{4} \in \mathbb{C}^{\left(m-t_{A}\right) \times t_{A}}$, $\left(B_{2}\right)_{2} \in \mathbb{C}^{\left(m-t_{A}-t\right) \times\left(n-t_{A}-t\right)}$, and $\left(W_{2}^{A}\right)_{2} \in \mathbb{C}^{\left(n-t_{A}-t\right) \times\left(m-t_{A}-t\right)}$ satisfying

$$
\begin{gathered}
A=P\left(A_{1} \oplus A_{2}\right) Q^{-1}, \quad B=P\left[\begin{array}{cc}
A_{1} & O \\
B_{4} & \left(B_{2}\right)_{1} \oplus\left(B_{2}\right)_{2}
\end{array}\right] Q^{-1}, \\
W=Q\left(W_{1}^{A} \oplus\left(\left(W_{2}^{A}\right)_{1} \oplus\left(W_{2}^{A}\right)_{2}\right)\right) P^{-1},
\end{gathered}
$$

where $\left(\left(W_{2}^{A}\right)_{1} \oplus\left(W_{2}^{A}\right)_{2}\right) A_{2}, A_{2}\left(\left(W_{2}^{A}\right)_{1} \oplus\left(W_{2}^{A}\right)_{2}\right),\left(W_{2}^{A}\right)_{2}\left(B_{2}\right)_{2}$, and $\left(B_{2}\right)_{2}\left(W_{2}^{A}\right)_{2}$ are nilpotent matrices and $\left(\left(W_{2}^{A}\right)_{1} \oplus\left(W_{2}^{A}\right)_{2}\right) B_{4}=O$.

Proof. It follows from the fact that $A \preceq^{d, W, \ell} B$ is equivalent to $A^{*} \preceq^{d, W^{*}, r} B^{*}$ and after application Theorem 2.1.

While in Theorem 2.2 we use the same matrix names as in Theorem 2.1, we remark that they are not necessarily the same ones. That is, for example, matrix $P$ in Theorem 2.1 may be different from matrix $P$ in Theorem 2.2.

Theorem 2.3 Let $W \in \mathbb{C}^{n \times m}$ be a nonzero matrix and $A, B \in \mathbb{C}^{m \times n}$. The following conditions are equivalent:

(a) $A \preceq^{d, W} B$.

(b) $(A W)^{D}(A W)=(A W)^{D}(B W)=(B W)(A W)^{D}$ and $(W A)^{D}(W A)=(W A)^{D}(W B)=$ $(W B)(W A)^{D}$.

(c) $(A W)^{k_{1}}(B W)=(B W)(A W)^{k_{1}}=(A W)^{k_{1}+1}$ and $(W A)^{k_{2}}(W B)=(W B)(W A)^{k_{2}}=$ $(W A)^{k_{2}+1}$, where $k_{1}=\operatorname{ind}(A W)$ and $k_{2}=\operatorname{ind}(W A)$. 
(d) There exist nonsingular matrices $P \in \mathbb{C}^{m \times m}, Q \in \mathbb{C}^{n \times n}, A_{1}, W_{1}^{A} \in \mathbb{C}^{t_{A} \times t_{A}}$, and $\left(B_{2}\right)_{1},\left(W_{2}^{A}\right)_{1} \in \mathbb{C}^{t \times t}$, and there exist matrices $A_{2} \in \mathbb{C}^{\left(m-t_{A}\right) \times\left(n-t_{A}\right)},\left(B_{2}\right)_{2} \in \mathbb{C}^{\left(m-t_{A}-t\right) \times\left(n-t_{A}-t\right)}$, and $\left(W_{2}^{A}\right)_{2} \in \mathbb{C}^{\left(n-t_{A}-t\right) \times\left(m-t_{A}-t\right)}$ satisfying

$$
\begin{gathered}
A=P\left(A_{1} \oplus A_{2}\right) Q^{-1}, \quad B=P\left(A_{1} \oplus\left(\left(B_{2}\right)_{1} \oplus\left(B_{2}\right)_{2}\right)\right) Q^{-1}, \\
W=Q\left(W_{1}^{A} \oplus\left(\left(W_{2}^{A}\right)_{1} \oplus\left(W_{2}^{A}\right)_{2}\right)\right) P^{-1}
\end{gathered}
$$

where $\left(\left(W_{2}^{A}\right)_{1} \oplus\left(W_{2}^{A}\right)_{2}\right) A_{2}, A_{2}\left(\left(W_{2}^{A}\right)_{1} \oplus\left(W_{2}^{A}\right)_{2}\right),\left(W_{2}^{A}\right)_{2}\left(B_{2}\right)_{2}$, and $\left(B_{2}\right)_{2}\left(W_{2}^{A}\right)_{2}$ are nilpotent.

Proof. Suppose that $A, B \in \mathbb{C}^{m \times n}$ satisfy $A \preceq^{d, W} B$. This is, $A \preceq^{d, W, r} B$ and $A \preceq^{d, W, \ell} B$. By Theorem 2.1,

$$
\begin{gathered}
A=P\left(A_{1} \oplus A_{2}\right) Q^{-1}, \quad B=P\left[\begin{array}{cc}
A_{1} & B_{3} \\
O & \left(B_{2}\right)_{1} \oplus\left(B_{2}\right)_{2}
\end{array}\right] Q^{-1}, \\
W=Q\left(W_{1}^{A} \oplus\left(\left(W_{2}^{A}\right)_{1} \oplus\left(W_{2}^{A}\right)_{2}\right)\right) P^{-1},
\end{gathered}
$$

where all the blocks satisfy the conditions found in item (d) of Theorem 2.1. Using that $W A \preceq^{d} W B$ and making some computations we get $B_{3}=O$. This shows $(\mathrm{a}) \Longrightarrow(\mathrm{d})$. The remaining implications follow directly from Theorem 2.1 and Theorem 2.2.

For a rectangular matrix $A$ and a weight $W$ of adequate sizes, Castro-González and Vélez-Cerrada considered in [4] the $W$-support idempotent $A^{\sigma, W}=A(W A)^{D}=(A W)^{D} A$. The authors established conditions under which the projectors $A^{\sigma, W} W$ and $B^{\sigma, W} W$ coincide. Similarly, for the projectors $W A^{\sigma, W}$ and $W B^{\sigma, W}$. In addition, they characterized matrices $B$ such that $A^{\sigma, W}=B^{\sigma, W}$. We reconcile results in Theorems 2.1, 2.2 and 2.3 above with Theorem 2.1, Theorem 2.4, and Corollary 2.7 in [4]. In order to do that, we first observe that if $A \preceq^{d, W, r} B$ then, from Theorem 2.1 above, we can write

$$
P^{-1} B Q=\left[\begin{array}{ccc}
A_{1} & X & Y \\
O & \left(B_{2}\right)_{1} & O \\
O & O & \left(B_{2}\right)_{2}
\end{array}\right]
$$


where $B_{3}$ has been partitioned as $\left[\begin{array}{ll}X & Y\end{array}\right]$ according to the blocks of $\left(B_{2}\right)_{1} \oplus\left(B_{2}\right)_{2}$. Clearly, the matrix

$$
\widetilde{B}=\left[\begin{array}{cc}
A_{1} & X \\
O & \left(B_{2}\right)_{1}
\end{array}\right]
$$

is nonsingular and setting $\tilde{Y}=\left[\begin{array}{ll}Y^{*} & O\end{array}\right]^{*}$ we get

$$
B=P\left[\begin{array}{cc}
\widetilde{B} & \widetilde{Y} \\
O & \left(B_{2}\right)_{2}
\end{array}\right] Q^{-1}
$$

Since $B_{3}\left(\left(W_{2}^{A}\right)_{1} \oplus\left(W_{2}^{A}\right)_{2}\right)=O$, some algebraic manipulations yields $X=O$ and $\tilde{Y}\left(W_{2}^{A}\right)_{2}=$ $O$. So, if $A \preceq^{d, W, r} B$ then $B$ can be written as in (4) where $\widetilde{B} \in \mathbb{C}^{\left(t_{A}+t\right) \times\left(t_{A}+t\right)}$ is nonsingular, $\tilde{Y}\left(W_{2}^{A}\right)_{2}=O$, and $\left(B_{2}\right)_{2}\left(W_{2}^{A}\right)_{2}$ is nilpotent. Nevertheless, we remark that the matrix $B_{1}$ in [4, Theorem 2.1 (ii)] and the matrix $A_{1}$ in [4, Lemma 1.1] have the same size. This shows that conditions in our Theorem 2.1 does not imply those conditions in [4, Theorem 2.1]. That is, Theorem 2.1 above is essentially different from [4, Theorem 2.1]. The same occurs with Theorem 2.2 and [4, Theorem 2.4] and also with Theorem 2.3 and [4, Corollary 2.7]. However, it follows that

$$
A^{\sigma, W} W=B^{\sigma, W} W \quad \text { implies } \quad A \preceq^{d, W, r} B \text { and } B \preceq^{d, W, r} A \text {. }
$$

We can get similar results for left and both sides relations as well. The converse of this result is also true as we show in the following theorem.

Theorem 2.4 Let $W \in \mathbb{C}^{n \times m}$ be a nonzero matrix and $A, B \in \mathbb{C}^{m \times n}$.

(a) If $A \preceq^{d, W, r} B$ and $B \preceq^{d, W, r} A$ then $A^{\sigma, W} W=B^{\sigma, W} W$.

(b) If $A \preceq^{d, W, \ell} B$ and $B \preceq^{d, W, \ell} A$ then $W A^{\sigma, W}=W B^{\sigma, W}$.

(c) If $A \preceq^{d, W} B$ and $B \preceq^{d, W} A$ then $A^{\sigma, W}=B^{\sigma, W}$.

Proof. It is enough to prove only item (a) because the second one can be obtained in a similar way and the third one is immediate from [4]. In fact, from $A \preceq^{d, W, r} B$ and Theorem 2.1 we have that

$$
A=P\left(A_{1} \oplus A_{2}\right) Q^{-1}, \quad B=P\left[\begin{array}{cc}
A_{1} & B_{3} \\
O & \left(B_{2}\right)_{1} \oplus\left(B_{2}\right)_{2}
\end{array}\right] Q^{-1},
$$




$$
W=Q\left(W_{1}^{A} \oplus\left(\left(W_{2}^{A}\right)_{1} \oplus\left(W_{2}^{A}\right)_{2}\right)\right) P^{-1},
$$

where $A_{1} W_{1}^{A}$ and $\left(B_{2}\right)_{1}\left(W_{1}^{A}\right)_{1}$ are nonsingular, $\left(B_{2}\right)_{2}\left(W_{2}^{A}\right)_{2},\left(W_{2}^{A}\right)_{2}\left(B_{2}\right)_{2}, A_{2}\left(\left(W_{2}^{A}\right)_{1} \oplus\right.$ $\left.\left(W_{2}^{A}\right)_{2}\right)$ and $\left(\left(W_{2}^{A}\right)_{1} \oplus\left(W_{2}^{A}\right)_{2}\right) A_{2}$ are nilpotent matrices and $B_{3}\left(\left(W_{2}^{A}\right)_{1} \oplus\left(W_{2}^{A}\right)_{2}\right)=O$. It then follows that $B W=P\left(A_{1} W_{1}^{A} \oplus\left(\left(B_{2}\right)_{1}\left(W_{1}^{A}\right)_{1} \oplus\left(B_{2}\right)_{2}\left(W_{2}^{A}\right)_{2}\right)\right) P^{-1}$. Thus, $(B W)^{D}=$ $P\left(\left(A_{1} W_{1}^{A}\right)^{-1} \oplus\left(\left(\left(B_{2}\right)_{1}\left(W_{1}^{A}\right)_{1}\right)^{-1} \oplus O\right)\right) P^{-1}$ leads to $B W(B W)^{D}=P\left(I_{t_{A}} \oplus\left(I_{t} \oplus O\right)\right) P^{-1}$.

If we now partition

$$
A_{2}=\left[\begin{array}{ll}
\left(A_{2}\right)_{1} & \left(A_{2}\right)_{3} \\
\left(A_{2}\right)_{4} & \left(A_{2}\right)_{2}
\end{array}\right]
$$

we can get

$$
A_{2}\left(\left(W_{2}^{A}\right)_{1} \oplus\left(W_{2}^{A}\right)_{2}\right)=\left[\begin{array}{cc}
\left(A_{2}\right)_{1}\left(W_{2}^{A}\right)_{1} & \left(A_{2}\right)_{3}\left(W_{2}^{A}\right)_{2} \\
\left(A_{2}\right)_{4}\left(W_{2}^{A}\right)_{1} & \left(A_{2}\right)_{2}\left(W_{2}^{A}\right)_{2}
\end{array}\right] .
$$

Using that $B \preceq^{d, W, r} A$, we have that $(B W)^{D} B W=(B W)^{D} A W=A W(B W)^{D}$ and replacing by the expressions of $B W,(B W)^{D}$, and $A W$ it can be easily obtained that $\left(A_{2}\right)_{1}\left(W_{2}^{A}\right)_{1}=\left(B_{2}\right)_{1}\left(W_{1}^{A}\right)_{1},\left(A_{2}\right)_{3}\left(W_{2}^{A}\right)_{2}=O$, and $\left(A_{2}\right)_{4}=O$. Hence, the nilpotent matrix $A_{2}\left(\left(W_{2}^{A}\right)_{1} \oplus\left(W_{2}^{A}\right)_{2}\right)=\left(B_{2}\right)_{1}\left(W_{1}^{A}\right)_{1} \oplus\left(A_{2}\right)_{2}\left(W_{2}^{A}\right)_{2}$. Since $\left(B_{2}\right)_{1}\left(W_{1}^{A}\right)_{1}$ is nonsingular, $\left(B_{2}\right)_{1}$ must be absent in the decomposition of matrix $B$. So, by [4, Theorem 2.1], $A^{\sigma, W} W=B^{\sigma, W} W$.

From Theorem 2.1 (d), it follows directly that $A \preceq^{d, W, r} B$ implies $A^{\sigma, W} \preceq^{d, W, r} B^{\sigma, W}$. Analogously, for left and both sides relations similar implications can be stated.

We close this section emphasizing that the relations $\preceq^{d, W, r}, \preceq^{d, W, \ell}$, and $\preceq^{d, W}$ are pairwise different. It is enough to show that $A \preceq^{d, W, r} B$ does not imply $A \preceq^{d, W, \ell} B$. In fact, matrices $A, B$, and $W$ given in Example 2.1 satisfy $A \preceq^{d, W, r} B$ and $A \npreceq^{d, W, \ell} B$.

\section{Equal Weighted Drazin Projectors}

We recall that a square matrix $A$ is said to be $E P$ if $A A^{\dagger}=A^{\dagger} A$, where $A^{\dagger}$ denotes the Moore-Penrose inverse of $A$ (that is, $A A^{\dagger} A=A, A^{\dagger} A A^{\dagger}=A^{\dagger},\left(A A^{\dagger}\right)^{*}=A A^{\dagger}$, and $\left(A^{\dagger} A\right)^{*}=A^{\dagger} A$ hold $)$. These and similar matrices have been widely studied in different environments $[5,6,9,10]$.

Let $W \in \mathbb{C}^{n \times m}$. We now observe that, if $A \in \mathbb{C}^{m \times n}$, the matrices $(A W)^{D} A W$ and $(W A)^{D} W A$ are projectors of size $m \times m$ and $n \times n$, respectively. The following definition 
considers the case where both weighted Drazin projectors are equal and is inspired in the definition of EP matrix.

Definition 3.1 Let $W \in \mathbb{C}^{n \times n}$ be a nonzero matrix. A matrix $A \in \mathbb{C}^{n \times n}$ is called $E D P_{W}$ if satisfies $(A W)^{D} A W=(W A)^{D} W A$.

The class of all $E D P_{W}$ matrices will be denoted by $\mathcal{E D} \mathcal{P}_{W}$. Notice that if $A \in \mathcal{E D} \mathcal{P}_{W}$ then $P A P^{-1} \in \mathcal{E D} \mathcal{P}_{P W P^{-1}}$ for all nonsingular $P \in \mathbb{C}^{n \times n}$ because $\left(P A P^{-1}\right)^{D}=P A^{D} P^{-1}$. Our next aim is to characterize $E D P_{W}$ matrices.

Theorem 3.1 Let $A, W \in \mathbb{C}^{n \times n}$ with $W \neq O$. The following conditions are equivalent:

(a) $A \in \mathcal{E D} \mathcal{P}_{W}$

(b) There exist nonsingular matrices $P \in \mathbb{C}^{n \times n}, A_{1}, W_{1} \in \mathbb{C}^{t \times t}$, and there exist matrices $A_{2}, W_{2} \in \mathbb{C}^{(n-t) \times(n-t)}$ such that

$$
A=P\left(A_{1} \oplus A_{2}\right) P^{-1} \quad \text { and } \quad W=P\left(W_{1} \oplus W_{2}\right) P^{-1}
$$

where $A_{2} W_{2}$ and $W_{2} A_{2}$ are nilpotent.

Proof. Assume that $A \in \mathbb{C}^{n \times n}$ is $E D P_{W}$. Since $W \neq O$, we can write

$$
A=P\left(A_{1}^{\prime} \oplus A_{2}^{\prime}\right) Q^{-1} \quad \text { and } \quad W=Q\left(W_{1}^{\prime} \oplus W_{2}^{\prime}\right) P^{-1}
$$

where the block matrices have the properties indicated in Theorem 1.1. It is easy to see that

$$
(A W)^{D} A W=P\left(I_{t} \oplus O\right) P^{-1}, \quad \text { and } \quad(W A)^{D} W A=Q\left(I_{t} \oplus O\right) Q^{-1}
$$

Equating and partitioning

$$
P^{-1} Q=\left[\begin{array}{cc}
M & N \\
R & S
\end{array}\right]
$$

according to the blocks of $A$ we get $N=O$ and $R=O$, which implies that $Q=P(M \oplus S)$. The result follows by replacing $Q$ in the expression (5) of $A$ and $W$ and setting $A_{1}=$ $A_{1}^{\prime} M^{-1}, A_{2}=A_{2}^{\prime} S^{-1}, W_{1}=M W_{1}^{\prime}$ and $W_{2}=S W_{2}^{\prime}$. The converse is trivial.

We now study the pre-order $\preceq^{d, W}$ on the class of matrices with equal weighted Drazin projectors. 
Theorem 3.2 Let $W \in \mathbb{C}^{n \times n}$ be a nonzero matrix and $A \in \mathcal{E D P}_{W}$. The following conditions are equivalent:

(a) There exists $B \in \mathcal{E D P}_{W}$ such that $A \preceq{ }^{d, W} B$.

(b) There exist nonsingular matrices $V \in \mathbb{C}^{n \times n}, A_{1}, W_{1}, \in \mathbb{C}^{t \times t}$, and $\left(B_{2}\right)_{1},\left(W_{2}\right)_{1} \in \mathbb{C}^{r \times r}$, and matrices $A_{2} \in \mathbb{C}^{(n-t) \times(n-t)},\left(B_{2}\right)_{2},\left(W_{2}\right)_{2} \in \mathbb{C}^{(n-t-r) \times(n-t-r)}$ such that

$$
A=V\left(A_{1} \oplus A_{2}\right) V^{-1}, \quad B=V\left(A_{1} \oplus\left(\left(B_{2}\right)_{1} \oplus\left(B_{2}\right)_{2}\right)\right) V^{-1}
$$

and

$$
W=V\left(W_{1} \oplus\left(\left(W_{2}\right)_{1} \oplus\left(W_{2}\right)_{2}\right)\right) V^{-1},
$$

where $\left(B_{2}\right)_{2}\left(W_{2}\right)_{2},\left(W_{2}\right)_{2}\left(B_{2}\right)_{2}, A_{2}\left(\left(W_{2}\right)_{1} \oplus\left(W_{2}\right)_{2}\right)$, and $\left(\left(W_{2}\right)_{1} \oplus\left(W_{2}\right)_{2}\right) A_{2}$ are nilpotent and $\left(B_{2}\right)_{2} \in \mathcal{E D P} \mathcal{P}_{\left(W_{2}\right)_{2}}$.

Proof. Since $A \in \mathcal{E D} \mathcal{P}_{W}$, applying Theorem 3.1 we can write

$$
A=P\left(A_{1} \oplus A_{2}^{\prime}\right) P^{-1} \quad \text { and } \quad W=P\left(W_{1} \oplus W_{2}^{\prime}\right) P^{-1}
$$

where the block matrices have the properties indicated there. Partition

$$
B=P\left[\begin{array}{ll}
B_{1} & B_{3} \\
B_{4} & B_{2}
\end{array}\right] P^{-1}
$$

with the blocks of appropriate sizes accordingly to $A$. The equalities $(A W)^{D} A W=$ $B W(A W)^{D}$ and $(W A)^{D} W A=(W A)^{D} W B$ are equivalent to $B_{1}=A_{1}, B_{4}=O$ and $B_{3}=O$. So,

$$
B=P\left(A_{1} \oplus B_{2}\right) P^{-1} .
$$

Now, $B \in \mathcal{E D P}_{W}$ if and only if the equality $(B W)^{D} B W=(W B)^{D} W B$ holds, from we have $B_{2} \in \mathcal{E D P}_{W_{2}^{\prime}}$. Again, Theorem 3.1 applied to the matrix $B_{2}$ and the weight $W_{2}^{\prime}$ asserts that $B_{2}=P_{1}\left(\left(B_{2}\right)_{1} \oplus\left(B_{2}\right)_{2}\right) P_{1}^{-1}$ and $W_{2}^{\prime}=P_{1}\left(\left(W_{2}\right)_{1} \oplus\left(W_{2}\right)_{2}\right) P_{1}^{-1}$, where $\left(B_{2}\right)_{2}\left(W_{2}\right)_{2}$ and $\left(W_{2}\right)_{2}\left(B_{2}\right)_{2}$ are nilpotent matrices.

Setting $V=P\left(I_{t} \oplus P_{1}\right), A_{2}=P_{1}^{-1} A_{2}^{\prime} P_{1}$ and replacing in expressions (8) and (9) we obtain matrices $A$ and $B$ of (6), and matrix $W$ of (7). Moreover, $\left(B_{2}\right)_{2} \in \mathcal{E D} \mathcal{P}_{\left(W_{2}\right)_{2}}$ and $A_{2}\left(\left(W_{2}\right)_{1} \oplus\left(W_{2}\right)_{2}\right)$ and $\left(\left(W_{2}\right)_{1} \oplus\left(W_{2}\right)_{2}\right) A_{2}$ are nilpotent.Hence, (a) $\Longrightarrow(\mathrm{b})$ holds. The 
converse is trivial.

Of course that, right and left relations can also be analyzed on the class of matrices with equal weighted Drazin projectors obtaining similar results.

\section{Adjacent matrices under the $\preceq^{d, W, r}, \preceq^{d, W, \ell}$, and $\preceq^{d, W}$ relations}

For two given matrices $A, B \in \mathbb{C}^{m \times n}$, it is said that $A$ and $B$ are adjacents if $\operatorname{rank}(B-A)=$ 1 (see, for example, [7, 11]). In what follows, we investigate the expressions for two matrices to be adjacent under $\preceq^{d, W, r}, \preceq^{d, W, \ell}$, and $\preceq^{d, W}$ relations.

Theorem 4.1 Let $W \in \mathbb{C}^{n \times m}$ be a nonzero matrix and $A, B \in \mathbb{C}^{m \times n}$ such that $A \preceq d, W, r$ B. The following conditions are equivalent:

(a) $A$ and $B$ are adjacent.

(b) There exist nonsingular matrices $P \in \mathbb{C}^{m \times m}$, and $Q \in \mathbb{C}^{n \times n}$, and nonzero vectors $u \in \mathbb{C}^{m \times 1}$ and $v \in \mathbb{C}^{\left(n-t_{A}\right) \times 1}$ such that

$$
B=A+P\left[O \quad u v^{*}\right] Q^{-1}
$$

Proof. Let $A, B \in \mathbb{C}^{m \times n}$ with $A \preceq^{d, W, r} B$. From Theorem 2.1,

$$
A=P\left(A_{1} \oplus A_{2}\right) Q^{-1} \quad \text { and } \quad B=P\left[\begin{array}{cc}
A_{1} & B_{3} \\
O & \left(B_{2}\right)_{1} \oplus\left(B_{2}\right)_{2}
\end{array}\right] Q^{-1} \text {. }
$$

It can be shown that $\operatorname{rank}(B-A)=1$ if and only if

$$
\operatorname{rank}\left(\left[\begin{array}{c}
B_{3} \\
\left(\left(B_{2}\right)_{1} \oplus\left(B_{2}\right)_{2}\right)-A_{2}
\end{array}\right]\right)=1
$$

which is equivalent to

$$
\left[\begin{array}{c}
B_{3} \\
\left(B_{2}\right)_{1} \oplus\left(B_{2}\right)_{2}
\end{array}\right]=\left[\begin{array}{l}
O \\
A_{2}
\end{array}\right]+u v^{*}
$$


for some nonzero vectors $u \in \mathbb{C}^{m \times 1}$ and $v \in \mathbb{C}^{\left(n-t_{A}\right) \times 1}$. Replacing in the expression above we have $B=A+P\left[\begin{array}{ll}O & u v^{*}\end{array}\right] Q^{-1}$. Observe that $A \neq B$ in item (a) is equivalent to $u \neq 0$ and $v \neq 0$ in item (b).

Analogously, we can give the following similar results.

Theorem 4.2 Let $W \in \mathbb{C}^{n \times m}$ be a nonzero matrix and $A, B \in \mathbb{C}^{m \times n}$ such that $A \preceq d, W, \ell$ B. The following conditions are equivalent:

(a) $A$ and $B$ are adjacent.

(b) There exist nonsingular matrices $P \in \mathbb{C}^{m \times m}, Q \in \mathbb{C}^{n \times n}$, and nonzero vectors $u \in$ $\mathbb{C}^{\left(m-t_{A}\right) \times 1}$ and $v \in \mathbb{C}^{n \times 1}$ such that

$$
B=A+P\left[\begin{array}{c}
O \\
u v^{*}
\end{array}\right] Q^{-1}
$$

Theorem 4.3 Let $W \in \mathbb{C}^{n \times m}$ be a nonzero matrix and $A, B \in \mathbb{C}^{m \times n}$ such that $A \preceq^{d, W} B$. The following conditions are equivalent:

(a) $A$ and $B$ are adjacent.

(b) There exist nonsingular matrices $P \in \mathbb{C}^{m \times m}, Q \in \mathbb{C}^{n \times n}$, and nonzero vectors $u \in$ $\mathbb{C}^{\left(m-t_{A}\right) \times 1}$ and $v \in \mathbb{C}^{\left(n-t_{A}\right) \times 1}$ such that

$$
B=A+P\left(O \oplus u v^{*}\right) Q^{-1}
$$

Remark 4.1 If $A$ and $B$ are adjacent matrices then

(i) either $A \preceq^{d, W, r} B$ and $B \preceq^{d, W, r} A$ hold, or $A W$ and $B W$ are adjacent matrices,

(ii) either $A \preceq^{d, W, \ell} B$ and $B \preceq^{d, W, \ell} A$ hold, or $W A$ and $W B$ are adjacent matrices.

Indeed, in case (i), $\operatorname{rank}(B W-A W) \leq \operatorname{rank}(B-A)=1$ if $A$ and $B$ are adjacent. That is, either $A W=B W$ holds or $A W$ and $B W$ are adjacent matrices. Similarly for the case (ii). 


\section{Further considerations on weighted partial orders}

For a nonzero matrix $W \in \mathbb{C}^{n \times m}$, we now consider the set

$$
\mathcal{M}_{W, r}=\left\{A \in \mathbb{C}^{m \times n}: \operatorname{ind}(A W) \leq 1\right\}
$$

Observe that $\mathcal{M}_{W, r} \neq \emptyset$ since $\operatorname{rank}\left(\left(W^{*} W\right)^{2}\right)=\operatorname{rank}\left(\left(W^{*} W\right)\left(W^{*} W\right)^{*}\right)=\operatorname{rank}\left(W^{*} W\right)$ yields $\operatorname{ind}\left(W^{*} W\right) \leq 1$ and then $W^{*} \in \mathcal{M}_{W, r}$.

Definition 5.1 Let $A, B \in \mathcal{M}_{W, r}$. It is said that $A \preceq \preceq^{\#, r} B$ if $A W \leq^{\#} B W$.

We remark that the relation $\preceq \#, W, r$ is a pre-order and coincides with the restriction of $\preceq^{d, W, r}$ on $\mathcal{M}_{W, r}$. Moreover, we stand out that the representation Theorem 2.1 is also true for the relation in Definition 5.1. In addition, taking into account that $A \in \mathcal{M}_{W, r}$, it then holds that either $A_{2} W_{2}=O$ or $A_{2} W_{2}$ is absent. Furthermore, since $k_{1} \in\{0,1\}$ and $\left|k_{1}-k_{2}\right| \leq 1$, it results $k_{2} \in\{0,1,2\}$ by [13, Theorem 11.1.2]. Then, $W_{2} A_{2}$ is absent if $k_{2}=0, W_{2} A_{2}=O$ if $k_{2}=1$ or $\left(W_{2} A_{2}\right)^{2}=O \neq W_{2} A_{2}$ if $k_{2}=2$.

Since $\leq \#$ is a partial order on index at most one matrices in $\mathbb{C}^{m \times m}$, we establish the following result.

Theorem 5.1 The relation $\preceq \#, W, r$ is a partial order on $\mathcal{M}_{W, r}$ provided that $W$ has full row rank.

Next we consider the set $\mathcal{P}_{W, r}=\left\{\mathcal{Z} \subseteq \mathbb{C}^{m \times n}: \preceq^{d, W, r}\right.$ is a partial order on $\left.\mathcal{Z}\right\}$ ordered by set inclusion.

Theorem 5.2 If $W \in \mathbb{C}^{n \times m}$ has full row rank then $\mathcal{M}_{W, r}$ is a maximal element of $\mathcal{P}_{W, r}$.

Proof. We first observe that $\mathcal{M}_{W, r} \in \mathcal{P}_{W, r}$. Assume that there exists a subset $\mathcal{Z} \in \mathcal{P}_{W, r}$ such that $\mathcal{M}_{W, r} \subseteq \mathcal{Z}$. If we suppose that $A \in \mathcal{Z}-M_{W, r}$ then $\operatorname{ind}(A W)>1$ and Theorem 1.1 assures that $A=P\left(A_{1} \oplus A_{2}\right) Q^{-1}$ and $W=Q\left(W_{1} \oplus W_{2}\right) P^{-1}$ as indicated in (1). Since $\operatorname{ind}\left(A_{2} W_{2}\right)>1$, we get $A_{2} W_{2} \neq O$ [3]. On the other hand, set $B=P\left(A_{1} \oplus O\right) Q^{-1}$. It can easily be seen that $B \in \mathcal{M}_{W, r} \subseteq \mathcal{Z}$. By Theorem 2.1, $A \preceq^{d, W, r} B$ holds. Now, by definition, it is easy to that $B \preceq^{d, W, r} A$. Since $A, B \in \mathcal{Z}$ and $\preceq^{d, W, r}$ is antisymmetric on $\mathcal{Z}$, we get $A=B$. Hence, $A_{2}=O$, which is a contradiction. 
Similarly, if $A, B \in \mathcal{M}_{W, \ell}=\left\{A \in \mathbb{C}^{m \times n}: \operatorname{ind}(W A) \leq 1\right\}$, we define $A \preceq^{\#, W, \ell} B$ if $W A \leq \# W B$. It is obtained that $\preceq^{\#, W, \ell}$ is a partial order on $\mathcal{M}_{W, \ell}$ provided that $W$ has full column rank. It is also valid that $\mathcal{M}_{W, \ell}$ is a maximal element among all the subsets $\mathcal{Z}$ of $\mathbb{C}^{m \times n}$ satisfying that $\preceq^{d, W, \ell}$ is a partial order on $\mathcal{Z}$.

Finally, defining $A \preceq^{\#, W} B$ if $A \preceq^{\#, W, r} B$ and $A \preceq^{\#, W, \ell} B$ for $A, B \in \mathcal{M}_{W, r} \cap \mathcal{M}_{W, \ell}$, it can also be established that $\preceq \#, W$ is a partial order provided that $W$ has full rank.

\section{Acknowledgements}

This paper was partially supported by Universidad Nacional de La Pampa, Facultad de Ingeniería of Argentina (grant Resol. $N^{\circ}$ 049/11) and the third author was partially supported by Ministerio de Economía y Competitividad of Spain (grant DGI MTM201018228).

\section{References}

[1] J.K. Baksalary, J. Hauke, Inheriting independence and chi-squaredness under certain matrix orderings, Statistics and Probability Letters, 2 (1984), 35-38.

[2] J.K. Baksalary, J. Hauke, G.P.H. Styan, On some distributional properties of quadratic forms in normal variables and some associated matrix partial orderings, Multivariate Analysis and its Applications, 24 (1994), 111-121.

[3] S.L. Campbell, C.D. Meyer Jr., Generalized Inverse of Linear Transformations. Dover, New York, Second Edition, 1991.

[4] N. Castro-González, J.Y. Vélez-Cerrada, The weighted Drazin inverse of perturbed matrices with related support idempotents, Applied Mathematics and Computation, 187 (2007), 756-764.

[5] D.S. Djordjević, Y. Wei, Operators with equal projections related to their generalized inverses, Applied Mathematics \& Computations, 155, 3 (2004), 655-664. 
[6] J.J. Koliha, P. Patrício, Elements of rings with equal spectral idempotents, Journal of the Australian Mathematical Society, 72 (2002), 137-152.

[7] Li-Ping Huang, Zhe-Xian Wan, Geometry of skew-Hermitian matrices, Linear Algebra and its Applications, 396 (2005), 127-157.

[8] S. K. Mitra, P. Bhimasankaram, S. B. Malik, Matrix partial orders, shorted operators and applications. World Scientific Publishing Company, 2010.

[9] D. Mosić, D.S. Djordjević. Partial isometries and EP elements in rings with involution, Electronic Journal of Linear Algebra, 18 (2009), 761-772.

[10] P. Patrício, C. Mendes Araújo, Moore-Penrose invertibility in involutory rings: the case $a a^{\dagger}=b b^{\dagger}$, Linear and Multilinear Algebra, 58, 4 (2010), 445-452.

[11] P. Šemrl, A.R. Sourour, Adjacency preserving maps on hermitian matrices, Journal of the Australian Mathematical Society, 95, 1 (2013), 129-133.

[12] M. Tošić, D.S. Cvetković-Ilić, Invertibility of a linear combination of two matrices and partial orderings, Applied Mathematics and Computation, 218, 9 (2012), 4651-4657.

[13] G. Wang, Y. Wei, S. Qiao, Generalized Inverses: Theory and Computations, Science Press, Beijing/New York, 2004. 